\title{
Concept and Development of an Autonomous Wearable Micro-fluidic Platform for real time $\mathrm{pH}$ Sweat Analysis
}

\author{
Vincenzo F. Curto, S. Coyle, R. Byrne, N. Angelov, D. Diamond, F. Benito- \\ Lopez
}

CLARITY: Centre for Sensor Web Technologies, National Centre for Sensor Research, School of Chemical Sciences, Dublin City University, Dublin 9, IRELAND

\begin{abstract}
In this work the development of an autonomous, robust and wearable micro-fluidic platform capable of performing on-line analysis of $\mathrm{pH}$ in sweat is discussed. Through the means of an optical detection system based on a surface mount light emitting diode (SMD LED) and a light photo sensor as a detector, a wearable system was achieved in which real-time monitoring of sweat $\mathrm{pH}$ was performed during 55 minutes of cycling activity. We have shown how through systems engineering, integrating miniaturised electrical components, and by improving the micro-fluidic chip characteristics, the wearability, reliability and performance of the micro-fluidic platform was significantly improved.
\end{abstract}

Keywords: micro-fluidic; pH; SMD LED; light photo sensor; wearable system, sweat analysis

\section{Introduction}

Sweat is a body fluid naturally produced during physical exercise and emotional stress, and it is essentially a filtrate of blood plasma containing many substances such as sodium, chloride, potassium, bicarbonate, calcium, ammonia and organic compounds, such as glucose and lactate [1]. Sweating is primarily a mechanism of body thermoregulation to avoid dangerous rise in body temperature that correlates with the increases in metabolic rate of the individual. During this physiological process, electrolytes and liquid are excreted by sweat glands via a duct to the outer skin surface. [2] Through the analysis of its composition it is possible to obtain useful information regarding the physiological condition of the body and provides as well information about the health and well-being of the individual, especially during sport activities.

One of the key points that have to be taken into account when sweat analysis want to be carried out it is the way sweat is collected, especially during sport activities. Different sweat collection techniques have been employed over the pass years, including whole body wash down technique, where the whole body sweat loss is determined weighing the subject before and after exercise and all fluid lost is collected 
and stored.[3] Others sweat collection techniques involve the use of patches[4] and even a capsule configuration, made by a flexible adhesive membrane that is covered with an impermeable laboratory film paper, such as Parafilm.[5] However, these techniques are not able to give real-time information about the physical condition of the body, mainly due to a substantial sampling to analysis delay. Moreover, there is high risk of crosscontamination of the samples during sampling, handling and posterior analysis. Therefore, real-time sweat analysis when performed during exercise is a great challenge for sensor fabrication due to the need for on-body fluid handling, sensor deployment and data management. If all these issues can be accomplished, the obtained devices will be capable of provide immediate feedback of fluid loss and variations of sweat analytes, giving prompt and reliable information of athlete performance and/or general health.

Sweat analysis has been employed in the diagnosis of diseases [6], drugs abuse [7] and in the optimisation of the performance of athletes.[8] Using a gold disc microelectrode De Souza et. al. [9] develop a rapid and straightforward method for copper ions detection for clinical diagnosis of Wilson disease. Also, inorganic cations, amines and amino acids in human sweat were detected by capillary electrophoresis [10], while odour active compounds of human male armpit sweat, after fenugreek ingestion, were investigated by gas chromatography coupled to mass spectrometry and olfactometry [11]. Real-time measurements of sodium concentration in sweat was developed by Schazmann et. al. [12] using a sodium sensor belt (SSB) based on Ion Selective Electrodes (ISE). An application of this SSB was found for the diagnosis of Cystic Fibrosis (CF) disease.

In our laboratories we are interested in $\mathrm{pH}$ since it is a very important, but at the same time, easy to measure physiological parameter in sweat. Many research groups including us are studying ways of analysing $\mathrm{pH}$ in sweat. It was investigated before that there are several factors that correlate sweat $\mathrm{pH}$ with health. For instance, Patterson $e t$ al.[13] found a correlation between sweat $\mathrm{pH}$ and sodium and chloride concentration in 
sweat, suggesting that decreasing of sodium and chloride concentrations in sweat is followed by a decrease of its $\mathrm{pH}$. Furthermore, it was demonstrated that the ingestion of sodium bicarbonate led to the increase in blood and sweat pH's [14]. In addition, it was found that changes in the $\mathrm{pH}$ of the skin play a role in the pathogenesis of skin diseases, such as dermatitis and acne.[15]

Autonomous wearable sensors to monitor sport activities should consist of reliable systems capable of monitoring physical and/or bio-chemical conditions in real time so different factors need to be considered during the design of these systems. Firstly, sampling is crucial since sample needs to be collected and delivered to the sensing active area where a signal will be generated. A failure on this stage will compromise answer and reliability of the whole sensor. Moreover, other important requirements to take into account are: (1) low cost of manufacture, (2) affordable market price, (3) flexibility of the device in order to be wearable and adaptable to the body contours (minimising the discomfort to the wearer), (4) easy signal to wearer interface and (5) long term stability.

In our laboratories, as part of BIOTEX (Biosensing textile for health management) project http://www.biotex-eu.com/, the first generation of a wearable, wireless sweat analysis system was successfully fabricated and tested [16]. This sensor was integrated into a wearable platform consisting of a black masked case containing a fluid-handing system and an optical detector. A textile-based fluidic system was used to draw and deliver sweat to the sensing area where a $\mathrm{pH}$ sensitive dye was incorporated in one end of the polyester/lycra ${ }^{\circledR}$ blends wicking textile. The colorimetric response of the pH sensitive dye was detected using Light Emitted Diodes (LEDs) integrated and positioned over the fabric channel into the device holder. 
In this paper, we present the technological achievements in performing real-time $\mathrm{pH}$ sweat analysis in real time coming from the basic concept of the textile-based fluid handling device towards a miniaturised wearable micro-fluidic device. We focus our investigations in two main streams: the size reduction of the electronic components that compose the detection system of the device and a smart micro-fluidic chip design and fabrication, capable of improving sample collection, minimise dead volumes and so sample cross-contamination and decrease detection times. Following both approaches we were successful in the generation of a fully autonomous and functional wearable micro-fluidic platform for real-time sweat analysis.

\section{Experimental}

\subsection{Materials}

The fabrication of the micro-fluidic devices was carried out using a laser ablation system-excimer $/ \mathrm{CO}_{2}$ laser (Optec LaserMicromachining Systems, Belgium) and a thermal roller laminator (Titan-110, GBC Films, USA). Poly(methyl-methacrylate) (PMMA) $50 \mu \mathrm{m}$ slides were purchased from Goodfellow, UK. $80 \mu \mathrm{m}$ double-sided pressure sensitive adhesive film (PSA-AR8890) was obtained from Adhesives Research, Ireland.

Artificial sweat was prepared according to the standard ISO $3160-2(20 \mathrm{~g} / \mathrm{L} \mathrm{NaCl}, 17.5$ $\mathrm{g} / \mathrm{L} \mathrm{NH}_{4} \mathrm{OH}, 5 \mathrm{~g} / \mathrm{L}$ acetic acid and $15 \mathrm{~g} / \mathrm{L}$ lactic acid) (Sigma-Aldrich, St. Louis, USA). Bromocresol purple dye (BCP), hydrochloric acid, sodium hydroxyde and tetraoctyl ammonium bromide were obtained from Sigma Aldrich, St. Louis, USA. Cotton gauze was purchased from Boots Pharmaceuticals, Ireland.

Yellow surface mount LEDs (SMD LEDs) of peak wavelength 590nm, (KP 2012SYC, Kingbright) and surface mount light photo sensor modules (APDS-9004, Avago Technologies) were purchased from Farnell Ireland. Temperature sensors (Analog Devices, ADT 7301) and humidity sensors (Sensirion, SHT 11) were purchased from 
Radionincs. Arduino microcontrollers and Xbee wireless modules were purchased from Sparkfun Electronics, Boulder, Colorado, USA.

The in-house-designed case and micro-fluidic holder of wired and wireless detectors were fabricated using a 3D printer (Stratasys, USA) in acrylonitrile butadiene styrene co-polymer (ABS) plastic in order to protect the electronics from moisture and liquid and to minimise interferences from ambient light during the operation of the device. The printed parts were designed using ProEngineer CAD/CAM software package ${ }^{\circledR}$.

\subsection{Textile-based fluid handling device}

The textile-based fluid handling device used a fabric based fluidic system, where sweat entered and flowed through a channel by capillary action and collected by an absorbent patch at the end of the channel. The channel was created by coating regions of the fabric with hydrophobic materials. The channel width was $8 \mathrm{~mm}$ and overall length was over $30 \mathrm{~mm}$. This was designed to accommodate multiple sensors. A cover was held $5 \mathrm{~mm}$ above the fabric layer by means of a rubber gasket. The fabric patch covered an area of $40 \times 50 \mathrm{~mm} . \mathrm{pH}$ sensitive $\mathrm{BCP}$ dye was applied to the fabric channel and optical sensing components were positioned in the plastic cover. A paired emitterdetector LED configuration was used to measure the colour change of the fabric. Two red LED's $(\lambda=660 \mathrm{~nm})$ were positioned at an angle above the fabric in a reflectance mode configuration. Black silicone was placed around the sides of the detector LED to block ambient light (Fig. 3-a). The LEDs were chosen because they have a narrow viewing angle $\left(34^{\circ}\right)$ to help focus the light on a smaller sensing region. A minimum sensing region of $5 \times 5 \mathrm{~mm}$ was needed, based on the height and angles that the LEDs were positioned. As the fabric channel was $8 \mathrm{~mm}$ wide, based on the requirements of other sensors placed on the channel, the $\mathrm{pH}$ dye was printed onto a $8 \mathrm{x} 7 \mathrm{~mm}$ area. [17] 


\subsection{Micro-fluidic Platform}

All the micro-fluidic chips, Fig.1 a and c, were fabricated using multilayer lamination protocol. In brief, a $\mathrm{CO}_{2}$ laser system was used to cut the various polymer layers. Micro-fluidic channels were cut from $80 \mu \mathrm{m}$ thick layer of PSA and laminated onto PMMA $50 \mu \mathrm{m}$ thick, using the thermal roller laminator. Fig. 1-b shows the fabrication of the two generations of the micro-fluidic chips. The sensing area is a piece of nylon lycra ${ }^{\circledR}$ textile $(3 \times 3 \mathrm{~mm})$ embedded in the middle of the device with a $\mathrm{pH}$ sensitive dye, bromocresol purple (BCP), which varies colour according to the $\mathrm{pH}$ of the sweat. The immobilisation of $\mathrm{BCP}$ is performed in an ethanol solution containing tetraoctyl ammonium bromide to avoid leaching of the dye during the contact with aqueous solutions and sweat. $\mathrm{BCP}$ dye colour range varies in the physiological $\mathrm{pH}$ range of sweat ( $\mathrm{pH} 5-7)$.

\section{$<$ Fig. 1.>}

The first generation of the micro-fluidic chip consists on a small structure of 20 by $10 \mathrm{~mm}$, as shown in Figure 1-a. A round inlet of $2 \mathrm{~mm}$ in diameter is placed at the top of the channel that has an overall length of $8 \mathrm{~mm}$. The first part of the channel is characterised by a drop shape (length of $6 \mathrm{~mm}$ ) structure, which acts as a collector of fresh sweat coming from the skin. The collector is followed by a $2 \mathrm{~mm}$ length channel, while the channel following the sensing area (exhaustion channel) presents a length of 2 $\mathrm{mm}$. Both channels have a width and depth equal to $400 \mu \mathrm{m}$ and $80 \mu \mathrm{m}$, respectively. At the end of the channel a $5 \mathrm{~mm}$ diameter chamber contains the absorbent material $(4 \times 3$ $\mathrm{mm})$.

The design of the second generation of the micro-fluidic chip takes into consideration other parameters like long-term operability and flow control by the integration of a cotton thread inside the micro-channel to ensure a homogeneous sweat 
flow rate through the channels (Figure $1 \mathrm{~b}, \mathrm{c}$ ). The overall dimensions of the microfluidic chip are $45 \times 20 \mathrm{~mm}$. Inlet diameter, channel width, depth and collector are the same than in the first generation, while the exhaustion channel has a length of $4 \mathrm{~mm}$. The absorbent material has a diameter of $6 \mathrm{~mm}$ and it is placed into a round chamber of $12 \mathrm{~mm}$ in diameter.

\subsection{Detection system}

2.3.1 Textile-based fluid handling device

The LEDs were controlled by a central control unit that was supplied by CSEM. The detector LED was reverse-biased at $+5 \mathrm{~V}$, which charges the capacitance across it. This is discharged by the photocurrent generated upon incident light. The discharge rate is proportional to the intensity of the light reaching the detector. A digital output can be obtained by using a basic detection/timer circuit, which measures the time it takes the photocurrent to discharge the voltage from $+5 \mathrm{~V}(\operatorname{logic} 1)$ to $+1.7 \mathrm{~V}(\operatorname{logic} 0)$. Data was wirelessly transmitted to a laptop for analysis via Bluetooth.

\subsubsection{Wired device}

By reducing the fluidic channel from a width of $8 \mathrm{~mm}$ to $400 \mu \mathrm{m}$ and reducing the sensing region from $56 \mathrm{~mm}^{2}$ to $4 \mathrm{~mm}^{2}$ the optical detection components also needed to be miniaturised and be aligned to focus on a smaller region. For this reason superbright yellow SMD LEDs $(\lambda=590 \mathrm{~nm})$ were chosen.

\subsubsection{Wired System 1 (not shown here)}

The first miniaturised system used the same principle as the textile-based fluid handling device and a pair of SMD LEDs were placed above and below the sensing area of the microfluidic chip in a transmission configuration. In first trials black masking 
tape was used to position the SMD LEDs and also to block ambient light. One of the SMD LEDs acted as light source while the other was reverse biased and acted as a detector. A resistor was connected in series with the source SMD LED to attenuate the light to prevent saturation of the detector. Both SMD LEDs were controlled by a microcontroller platform, Lilypad Arduino, which is designed for wearable applications. The detector SMD LED was connected to the digital I/O pin of the Arduino and a timing routine was used to measure the discharge time from logic ' 1 ' to ' 0 '. Data was sampled at $2 \mathrm{~Hz}$ and transferred to a laptop by an RS232 serial link.

\subsubsection{Wired System 2}

In this system SMD LED was used as light source, while surface mount light photo sensor (APDS-9004, Avago Technologies) was chosen as a detector. The circuit diagram of the detector is shown in figure 2. The SMD LED intensity was controlled by a current limiting resistor $\left(\mathrm{R}_{1}\right) .30 \mathrm{awg}$ wire leads were soldered to the pins of the components and the devices subsequently coated in silicone (Dow Corning, Sylgard 184) to protect the components and also to prevent short circuits between the pins. The output pin of the light photo sensor module was connected to the analogue input channel of the microcontroller. A capacitor (C) was used as a low pass filter to remove high frequency noise, and a load resistor $\left(\mathrm{R}_{2}\right)$ was used to control the current to voltage output signal of the light photo sensor.

\section{$<$ Fig.2.>}

A transmission configuration was used, where the light passes directly through the sensing material. The SMD LED and light photo sensor were placed on either side of the chip. For the on-body trials a robust system was required to position the optical components onto the microfluidic chip. Plastic 3D printed case was designed to hold 
the components in place, as shown in Fig. 3-b. The data was sampled at $2 \mathrm{~Hz}$ using either an Arduino Lilypad or Arduino Pro and transferred to a laptop by an RS232 serial link.

\subsubsection{Wireless device}

In this system wired connection to the laptop was replaced with a wireless link. One option was to attach a Bluetooth ${ }^{\circledR}$ modem (BlueSMiRF silver) to the Arduino device. At the time of development a new Arduino Funnel IO was released, which presents an Xbee socket. This device provided a more compact solution rather than adding a separate modem to the existing configuration. There is also an option of a longer range using Xbee Pro modules and lower power consumption for shorter ranges compared to Bluetooth. However, this system requires an Xbee base-station to be connected to a laptop, while many devices would have Bluetooth capability in-built. At the end, an Xbee module was chosen.

The optical components and associated electronics were assembled in the same way as the previous wired system (Fig. 2). In addition, a temperature sensor and humidity sensor were integrated into the device to measure skin temperature and the humidity of the surrounding environment. Digital sensors were used as these could be easily configured using the Arduino FIO. A plastic holder was made using 3D printer to hold the temperature sensor (Analog Devices, ADT 7301), humidity sensor (Sensirion, SHT 11) sensors, SMD light photo sensor and LED and the $\mathrm{pH}$ microfluidic chip. A separate box was designed to hold the Arduino funnel, battery and control circuitry (Fig. 3-c).

\subsubsection{Portable device}


The portable device was designed to improve the wearability of the system by reducing the size of the overall design and customise a wireless system based on the sweat sensor requirements. The sampling point, sensing elements and associated electronics were combined into a single device, Fig. 3-d. The device once again uses a transmission mode configuration with a SMD LED and light photo sensor to detect colour changes related to $\mathrm{pH}$. A temperature sensor was included in the device to measure skin temperature. The wireless capability is based on the Tyndall $25 \mathrm{~mm}$ mote. This device operates in the $2.4 \mathrm{GHz}$ ISM Band and uses ZigBee protocols for communication. A base-station mote is connected to a laptop to receive the transmitted data and $\log$ this for analysis.

<Fig. 3.>

\section{Results and discussion}

\subsection{Detection system}

Colorimetric detection is performed as the sensing material changes colour according to the $\mathrm{pH}$ of sweat. A light source is needed to illuminate the fabric and an optical detector is needed to measure the transmitted or reflected light. In the textilebased fluid handling device, the optical sensing configuration used a reflectance measurement where the light source and the detector were positioned at an angle above the sensing layer, as shown in Fig. 4-a. This meant that an adequate sensing region (56 $\mathrm{mm}^{2}$ ) was needed to ensure accurate measurements, so that the detector LED would only receive light reflected from the sensing region of the fabric and not from the surrounding textile. This was important considering possible movement effects during wear. $3 \mathrm{~mm}$ LEDs were used and were encased in clear silicone to avoid the effects of 
sweat condensation. A reverse-biased LED was used as a detector. This arrangement used the inherent capacitance of the LED and measured, the discharge time using a microcontroller. The discharge rate is proportional to the intensity of the light reaching the detector.

Despite the good performance obtained by the textile-based fluid handling device, the bulkiness of the LED-LED system makes difficult to place and adapt the sensor in different parts of the body. Therefore, we decided to miniaturise the detection system by replacing the 3mm LEDs by a SMD LEDs. This reduced the bulkiness of the detection system considerably since the SMD LED is $1 \mathrm{x} 1 \mathrm{~mm}$ and flat $(500 \mu \mathrm{m})$ ensuring a more compact and compatible detector for wearable applications.

The first detector realised using SMD LED was the wired system 1, where a reverse biased SMD LED in transmittance was used as detector. During preliminary experiments this system demonstrated good response for changes in $\mathrm{pH}$ from $\mathrm{pH} 4$ to $\mathrm{pH}$ 7. However, the detection system did not have sufficient sensitivity when calibrations were performed using artificial sweat solutions between these $\mathrm{pH}$ ranges.[18] Therefore a SMD light photo sensor (APDS-9004, Avago Technologies) was chosen as detector, with the aim to increase its sensitivity respect the reverse biased SMD LED.

$$
\text { <Fig. 4.> }
$$

The SMD LED and light photo sensor were placed above and below the sensing area of the micro-fluidic chip in a tidy and compact structure as shown in Fig.4-b. The light emitted by the SMD LED is attenuated as it passes through the textile that presents different colour depending on the $\mathrm{pH}$ of the sample. This change in light intensity is sensed by the light photo sensor operating in photoconductive mode [19] where the $\mathrm{V}_{\text {out }}$ (Fig.2) is proportional to the photocurrent generated by the transmitted light. A black plastic holder for the wired system 2 was made using the $3 \mathrm{D}$ printer to align the SMD 
LED and the light photo sensor. Initially, the SMD LED and the light photo sensor were integrated in a wired configuration (Fig.4-b), wherein both were controlled by an Arduino Pro platform, which uses the ATmega168 microcontroller.

Therefore, in the second generation of the device the replacement of an Arduino Pro by an Arduino Funnel IO, which uses the ATmega328P and contains an Xbee socket, enabled a wireless system to be realised through which data was wirelessly transmitted to an Xbee base-station connected to a remote laptop via a USB serial link. With this configuration it was possible to provide more freedom of movements to the wearer and increasing the spatial operation of the system.

In spite of the good performance of the detector, its packaging was performed in such a way that it was too big and uncomfortable to carry during the exercise period. A more compact structure will provide a better adaptability of the whole system to the body contours. That is why we decided to work in the fabrication of a miniaturised system.

Fig.3-d shows the third generation of the wearable detector device, in which all the electronic components are integrated in a much smaller cordless platform (2.5 $\square \times 3$ $\mathrm{cm})$ capable of wireless transmission of $\mathrm{pH}$ sweat data directly to a laptop. This device is based on the Tyndall $25 \mathrm{~mm}$ mote, which is a modular stackable layer solution http://www.tyndall.ie/mai/.[20,21] This design opens the possibility of performing real-time sweat analysis, minimising the discomfort of the wearer while simultaneously providing information about the wearer's physiological conditions during exercise session. Moreover, due to its small dimensions, several devices could be used in different parts of the body simultaneously, to map $\mathrm{pH}$ sweat composition over the whole body.

\subsection{Micro-fluidic chip fabrication}


The employment of a micro-fluidic chip to perform sweat analysis gives several advantages over previous systems presented in literature.[3,17] For instance, in the textile-based fluid handling device it is employed a "fluid handling system" based on fabrics with inherent moisture wicking properties. Despite of the good performance and the inherent capability of the system to collect fresh sample over a period of time, the system needs high volume of liquid (milliliter) to carry out the analysis and activate the passive pump system prior use. These factors limit the continuous operability of the sensor, in fact a decrease in the sweat rate compromises its real-time operation.

To overcome these limitations, the development of micro-fluidic systems allows a substantially reduction of the volume that is needed to monitor certain analytes in sweat, i.e. $\mathrm{pH}$, because of the drastic reduction of channel width and length and therefore the sensing area. Moreover, since the sensing area is enclosed inside the micro-fluidic device cross-contamination from other skin areas and the surrounding environment is very unlikely.

In section 2.3 two generations of micro-fluidic chips were described, in where a the main advantage was the reduction of the sample volume to less than 10 $\mu 1 .[18]$ In both micro-fluidic chip configurations the sweat is drawn into the sensing area by an absorbent fiber placed at the end of the channel that acts as a passive pump system. Figure 5 shows four pump-less micro-fluidic devices and the relation of channel length with the time necessary for the fluid to reach the sensing area. The channel of $2 \mathrm{~mm}$ ensures fast sensor response reducing the delay time $\left(t_{d}\right)$ between the generation of the sweat in the skin and the sensor response to less than $20 \mathrm{~s}$.

$$
\text { <Fig. 5.> }
$$

Nevertheless, a better control of the flow rate in the microfluidic was obtained by the integration of a cotton thread inside the micro-channel. The use of cotton threads textiles into micro-fluidic systems has been explored by Li et al.[22], who demonstrated 
that using the intrinsic capillarity action of a thread textile it is possible to fabricate lowcost and easy-to-use devices for sensor purposes. Here the wicking property of a thread was used to transport sweat along the micro-fluidic channel, ensuring a homogeneous sweat flow rate, improving response time and platform robustness of the micro-fluidic device.

Although it was previously shown that the first generation of the micro-fluidic chip presents a good performance using a channel length of $2 \mathrm{~mm}$, in the second generation the length of the channel was increased seven times. The reason for this new design was mainly to ensure better flexibility and body adaptability of the inlet of the micro-fluidic chip when in contact with the skin with respect to the sensor electronics. A defined distance, see section 2.3, was necessary to avoid inlet detachment from the skin due to the tight position between micro-fluidic chip and electronics.

\subsection{Micro-fluidic chip performance}

\subsubsection{Passive pump}

Fig. 6 shows the autonomous pumping rate of the second generation of the microfluidic chip over time. The flow rate was calculated by adding a $10 \mu 1$ drop of DI water on the inlet of the micro-fluidic chip and recording the time the water is completely absorbed by the passive pump (absorbent). According to Morris et al.,[17] it is possible to define three operative regimes where the fluid rate is consistently different. When the system is in its dry state, the rate of fluid transports through the channel is due to the natural adsorption of the cotton thread placed into the channel. In our experiments is in the range of $1 \mu \mathrm{min}^{-1}$. Considering the volume of the micro-channel from the inlet to the sensing material (approximately $0.928 \mu \mathrm{l}$ ) and assuming a linear increment of the pumping rate during the first ten minutes, it has been calculated that the average time for the fluid to reach the sensing area is around 3 minutes. The second regime is characterised by an increasing of the pumping rate when the fluid reaches the super 
absorbent. The flow is controlled by the absorbing capacity of the absorbent material placed at the end of the channel. The pumping rate presents a maximum value of $2.37 \mu \mathrm{l}$ $\min ^{-1}$, after 20 minutes although the range of operation of this elevated flow can be defined between 10 and 40 minutes of operation, Fig. 6 . There is a quite large variation of the pumping rate over time according to the error shown in Figure 6, e.g. $\pm 1 \mu \mathrm{min}^{-1}$ for the worst scenario. The error is the average of four independent micro-fluidic chips and it can be attributed to the differences in the fabrication of the device since is manual. We have observed that the position of the thread in the channel is of extreme importance for the homogeneous flow behavior during testing.

Finally the last regime found during passive pumping starts when the absorbent gets saturated and so the flow rate slowed down considerably, stabilising a flow rate of around $0.5 \mu \mathrm{min}^{-1}$ till full absorbent saturation.

<Fig. 6.>

\subsubsection{Loading capacity of the micro-fluidic chip}

The loading capacity of the micro-fluidic chip, which is the maximum amount of sweat that the absorbent can uptake before the passive flow reaches zero, was calculated to be $68.7 \pm 6 \mu 1$. Therefore, the calculated life-time of the device, taking into account an average of sweat flow $\left(1.29 \pm 0.4 \mu \mathrm{min}^{-1}\right)$ was found to be around 53 minutes. Since in a healthy individual the sweating process starts between the 10-15 minute from the beginning of the sport activity [17], the whole operation time of the device can be approximately considered one hour in continuous mode. Moreover, due to the easy fabrication protocol of the device, multiple copies can be prepared in a single batch and replacement of an exhausted chip by a new fully operative device will increase the operation time. 
However, when longer life-times are required, a careful redesign of the micro-fluidic can be performed to extend the device operation time. For example, changing the amount of the absorbent material placed at the end of the channel, the second operative regime can be easily extended. Other option is, as well, to vary the channel dimensions to reduce the sweat flow rate.

These results suggested that the passive pump system is able to provide fresh sweat into the channel toward the sensing area without using any mechanical components, what it makes this device an effective low-cost pumping system for wearable applications.

\subsubsection{Sensor response}

The dynamic response of the sensing area of the micro-fluidic chip was investigated using solutions of different $\mathrm{pH}$ 's. A $40 \mu \mathrm{L}$ drop of $\mathrm{HCl}\left(\left[\mathrm{H}^{+}\right]=0.1 \mathrm{M}\right)$ and $\mathrm{NaOH}\left(\left[\mathrm{OH}^{-}\right]=0.01 \mathrm{M}\right)$ were alternatively placed in the inlet and they were let flow till the sensing area was reached, then the colour variation generated by the $\mathrm{pH}$ was recorded using the SMD LED-light photo sensor system.

Fig. 7 shows the response when the system is alternatively exposed to $\mathrm{pH} 1$ and 12 for four times. Briefly, low voltage detection of the light photo sensor corresponds to a less detecting light due to a darker colour of the sensing material. Because BCP dye changes colour from yellow (acidic form) to blue (basic form), a value of $\sim 0.8 \mathrm{~V}$ has to be interpreted as the high $\mathrm{pH}$, e.g. 12. Meanwhile, value of $\sim 2.35 \mathrm{~V}$ corresponds to acidic $\mathrm{pH}$.

<Fig. 7.>

The sensing area generates reproducible signals for both acidic and basic $\mathrm{pH}$ conditions. Two main parameters can be obtained from figure 7, the "delay time" and 
the "response time" summarised in Table 1.

The "delay time" $\left(t_{d}\right)$ corresponds to the time that sweat takes to reach the sensor area (textile), flat regions in Fig. 7.

The "response time" $\left(t_{\mathrm{r}}\right)$ corresponds to the time that sweat of certain $\mathrm{pH}$ fills the full sensing area and generates a stable signal in the detector, half parabolic regions in Fig. 7.

Table 1. Basic/acidic delay and response times of the micro-fluidic chip
\begin{tabular}{c|c|c|c|c} 
Basic $t_{\mathrm{d}} / \mathrm{min}$ & Acidic $\mathrm{t}_{\mathrm{d}} / \mathrm{min}$ & Basic $\mathrm{t}_{\mathrm{r}} / \mathrm{min}$ & Acidic $\mathrm{t}_{\mathrm{r}} / \mathrm{min}$ \\
\cline { 2 - 5 } Cycle 1 & 4.74 & 0.60 & 4.96 & 1.7 \\
Cycle 2 & 1.77 & 0.69 & 3.21 & 2.28 \\
Cycle 3 & 2.37 & 1.13 & 3.97 & 3.06 \\
Cycle 4 & 3.70 & 1.29 & 5.30 & 3.17
\end{tabular}

As observed in Table 1, both basic $t_{d}$ and $t_{r}$ in cycle 1 , showed substantial higher values than in the rest of the cycles. These results are in accordance with the pumping rate data presented in Fig. 6. In fact, when the system is on its first regime, the microfluidic chip is dry, the flow rate is slow because the passive pump system is not still active and the thread needs to hydrate. In the subsequent cycles acidic and basic $t_{d}$ are found to be lower than the first basic $t_{d}$ (cycle 1) but the values are progressively increasing with the number of cycles. This expected behaviour is following the trend of Fig. 6 where the pumping flow, once it has reached its maximum rate, starts to decrease gradually till regime three where the absorbent is completely full with sweat and the passive flow stops. It is interesting to point out that since concentrations of acid and base are not equal $\left(\mathrm{HCl}_{\text {sol, }}, 0.1 \mathrm{M}\right.$ and $\left.\mathrm{NaOH}_{\text {sol }} 0.01 \mathrm{M}\right)$ the sensor was able to get the two different $t_{d}$ coming from the acid and the basic solution. The acidic solution has a proton concentration ten times bigger than the hydroxide concentration of the basic solution, so neutralisation of the excess of acid in the textile needs to be performed 
before the sensor area starts to change colour and so to be observed by the SMD LEDlight photo sensor. In addition, experiments carried out with the same acidic and basic concentrations solutions showed that the $t_{d}$ is equal for a defined passive flow rate. The $t_{r}$ also presented the same trend than $t_{d}$ for each consecutive cycle. It is possible to observe that during the last basic $t_{r}$ the micro-fluidic chip is reaching the last regime where the passive pump flow is reduced significantly.

In short, these results confirm the reproducibility of the colorimetric detection during several cycles and the capability of the thread to draw the fluid toward the sensing area, generating a stable signal.

\subsubsection{Sensor calibration}

Figure 8 shows the calibration curve of the fabric textile containing the $\mathrm{pH}$ sensitive dye embedded as explained in section 2.1. The sensor material was tested using artificial sweat as standard solutions, where its $\mathrm{pH}$ was systematically varied from 1 to 14 adding an exact amount of $1 \mathrm{M} \mathrm{NaOH}$ solution. The textile exhibits a colour change from yellow to blue in this $\mathrm{pH}$ region and the change in colour was detected by the SMD LED-light photo sensor detector. The absorbed light is plotted as the inverse of the detected voltage. The experimental data are fit by a sigmoidal curve [17] following the eq.1.

$\mathrm{I}=\left[\mathrm{a} /\left(1+e^{\mathrm{b}(\mathrm{pH}-\mathrm{z})}\right)\right] * \mathrm{c}+\mathrm{d}$ eq. 1

where / is the detected light intensity, $a$ is the peak height, $b$ is the slope coefficient, $z$ is the point of inflection, $c$ the symmetry parameter for the sigmoid and $d$ accounts for a baseline offset.

The sensor presents a $\mathrm{pK}_{\mathrm{a}}$ of 6.8 which is slightly higher that the one from literature for the same dye in solution, $\mathrm{pK}_{\mathrm{a}}$ 6.2.[23] However, this effect has been previously 
observed by others concluding that immobilised dye in a solid support varies the $\mathrm{pK}_{\mathrm{a}}$ value due to a change of the microenvironment where the dye is immobilised.[24]

<Fig. 8.>

\subsubsection{On-body trial}

During on-body-trials the athlete was equipped with a Velcro belt located around the pelvis and the micro-fluidic device was fixed to the lumbar region of his back, as shown in the picture of Figure 9. The trail was carried out using the wireless detector (located in a pocket) and the micro-fluidic chip containing the thread. The $\mathrm{pH}$ of sweat was monitored in real-time for 55 minutes during a cycling trial. To verify the reliability of the measurements of the micro-fluidic device, a conventional glass $\mathrm{pH}$ electrode was used to carry out reference measurements.

<Fig. 9.>

In the trial the athlete worked at different loading intensity in particular light, moderate and high. During the warm-up period (between $0-10$ minutes) the athlete cycled in a moderate intensity mode for the first 5 minutes, then changes at high intensity regime with the aim to generate sweat as fast as possible. In this time interval it is possible to appreciate a drastic increase of the $\mathrm{pH}$, from 2.5 to 6.2 as shown in Fig. 9. Considering that the chip was placed on the body without any previous precondition, the first 5 minutes reading was generate by a dry chip, in its acidic state. After the initial 5 warm-up minutes the athlete started to sweat, therefore sweat is driven from the skin to the reservoir by the passive pump system that worked on its first regime (Fig. 6). 
From minutes 5 to 8 there is an increase in $\mathrm{pH}$ towards a stable value of 6.2. This reading is in accordance with the estimation made in section 3.3.1, where it was calculated that the average time for the fluid to reach the sensing area is around 3 minutes. Finally $6.2 \mathrm{pH}$ value is reached when fresh the sweat is covering the entire sensing area and a homogeneous colour was generated giving a stable signal.

In Fig. 9 it possible to appreciate two big drops of the $\mathrm{pH}$ value. During this time intervals the athlete changed alternatively the work intensity from high to moderate and it was observed a substantial increase on the sweat rate. The reason why these two big drops in $\mathrm{pH}$ value were generated can be attributed to physiology reasons, high sweat flow rate, variation of the $\mathrm{pH}$ value during the moderate exercise regime and an error occurred during the reading by the detector. At the best of our knowledge, we are not able to justify this $\mathrm{pH}$ drops so far; further investigations are been carried out in our laboratories to clarify this behaviour. In addition, the measurements made thorough a standard $\mathrm{pH}$ electrode showed a good correlation with the real-time $\mathrm{pH}$ data obtained using the micro-fluidic device.

The last $\mathrm{pH}$ meter reading value differs $0.5 \mathrm{pH}$ units with the $\mathrm{pH}$ electrode value. This can be attributed to a decreasing of the fluid flow rate in the micro-fluidic chip when the absorbent material started to be saturated and the system was working on the last regime.

\section{Conclusions}

In this work we have presented significant improvements made in the realisation of a fully autonomous wearable sensor capable of performing real-time chemical analysis of sweat composition, in particular $\mathrm{pH}$, during exercise events. The microfluidic system was developed using low-cost and flexible materials, and the passive pump system was developed through an absorbent material and a cotton thread inside the channel, improving robustness and dynamic response. Improvements in the 
detection system were gained through miniaturisation of its components as such detection system, wireless platform, battery and package. All these improvements have been made in order to have a platform that was physically compatible with the needs of wearable applications, while still providing a chemical analysis capability through the monitoring of reactive indicator colour ( $\mathrm{pH}$ in this case) using a SMD LED- light photo sensor detector.

Future work will be focused on the extension of the life-time of the micro-fluidic chip and on the use of the portable portable device and its possible integration in T-shirt, avoiding the use of external belt used for the placement of the whole system.

\section{Acknowledgements}

This work was supported by Science Foundation Ireland under grant 07/CE/I1 147 and a Research Career Start Programme 2010 fellowship from Dublin City University. Thanks to TEXSUS Spa (Italy) for supplying the CCAEP571LL absorbent material, Eng. Philip Angove and Mr. Javier Torres from Tyndall institute involved in the National Access Programme (NAP-251). Finally, Dr. Damien Maher who realised the $3 \mathrm{D}$ printing projects and work.

\section{References}

[1] A.G.R. Whitehouse, The Dissolved Constituents of Human Sweat, Proc. R. Soc. London, Ser. B, Biological Sciences 117 (1935) 139-154.

[2] K. Wilke, A. Martin, L. Terstegen, S.S. Biel, A short history of sweat gland biology, Int. J. Cosmet. Sci. 29 (2007)169-179.

[3] S.M. Shirreffs, R.J. Maughan, Whole body sweat collection in humans : an improved method with preliminary data on electrolyte content, J. Appl. Physiol. 82 (1997) 336-341.

[4] G. Hayden, H.C. Milne, M.J. Patterson, M.A. Nimmo, The reproducibility of closed-pouch sweat collection and thermoregulatory responses to exercise-heat stress, Eur. J. Appl. Physiol. 91 (2004) 748751 .

[5] G.R. Brisson, P. Boisvert, F. Peronnet, H. Perrault, D. Boisvert, J.S. Lafond, A simple and disposible sweat collector, Eur. J. Appl. Physiol. Occup. Physiol. 63 (1991) 269-272.

[6] L. Webster, H. Lochlin, Cystic fibrosis screening by sweat analysis: a critical review of techniques, Med J Aust. 1 (1977) 923-7.

[7] D.A. Kidwell, J.C. Holland, S. Athanaselis, Testing for drugs of abuse in saliva and sweat, J. Chromatogr. B 713 (1998) 111-135 
[8] R.J. Maughan, S.M. Shirreffs, Development of Individual Hydration Strategies for Athletes, Int. J. Sport Nutr. Exercise Metab. 18 (2008) 457-472

[9] A.P.R. d. Souza, A.S. Lima, M.O. Salles, A.N. Nascimento, M. Bertotti, The use of a gold disc microelectrode for the determination of copper in human sweat, Talanta 83 (2010) 167-170.

[10] T. Hirokawa, H. Okamoto, Y. Gosyo, T. Tsuda, A.R. Timerbaev, Simultaneous monitoring of inorganic cations, amines and amino acids in human sweat by capillary electrophoresis, Anal. Chim. Acta 581(2007) 83-88.

[11] R. Mebazaa, B. Rega, V. Camel, Analysis of human male armpit sweat after fenugreek ingestion: Characterisation of odour active compounds by gas chromatography coupled to mass spectrometry and olfactometry, Food Chem., 128 (2011) 227-235.

[12] B. Schazmann, D. Morris, C.Slater, S. Beirne, C. Fay, R. Reuveny, N Moyna, D. Diamond, A wearable electrochemical sensor for the real-time measurement of sweat sodium concentration, Anal. Methods 2 ( 2010) 342-348.

[13] M.J. Patterson, S.D.R. Galloway, M. A. Nimmo, Variations in regional sweat composition in normal human males, Experimental Physiology 85 (2000) 869-875.

[14] M.J. Patterson, S.D.R. Galloway, M.A. Nimmo, Effect of induced metabolic

alkalosis on sweat composition in men, Acta Physiol. Scand. 174 (2002) 41-46.

[15] M.H. Schmid-Wendtner, H.C. Korting, The pH of the Skin Surface and Its Impact on the Barrier Function, Skin Pharmacol. Physiol. 19 (2006) 296-302.

[16] S. Coyle, et al., BIOTEX - Biosensing textiles for personalised healthcare management, IEEE Trans. Inf. Technol. Biomed. 14 (2010) 364-370.

[17] D. Morris, S. Coyle, Y. Wu, K.T. Lau, G. Wallace, D. Diamond, Bio-sensing textile based patch with integrated optical detection system for sweat monitoring, Sens. Actuators B. 139 (2009) 231-236.

[18] F. Benito-Lopez, S. Coyle, R. Byrne, A.F. Smeaton, N.E. O'Connor, D. Diamond, Pump Less Wearable Microfluidic Device for Real Time pH Sweat Monitoring, Proc. Chem. 1(2009) 1103-1106.

[19] J. Fraden, Handbook of Modern Sensors - Physics, Design, and Applications, fourth ed., Springer, San Diego, 2010

[20] B. O'Flynn, S. Bellis, K. Mahmood, M. Morris, G. Duffy, K. Delaney, C. O’Mathuna, A 3-D Miniaturised Programmable Transceiver, Microelectron. Int., 22 (2005) 8-12.

[21] J. Barton, G. Hynes, B. O'Flynn, K. Aherne, A. Norman, A. Morrissey, 25mm sensor-actuator layer: A miniature, highly adaptable interface layer, Sens. Actuators A 132 (2006) 362-369.

[22] X. Li, J. Tian, W. Shen, Thread as a Versatile Material for Low-Cost Microfluidic Diagnostics, ACS Appl. Mater. Interface 2 (2010) 1-6.

[23] R.W. Sabnis, Handbook of Acid-Base Indicators, first ed., CRC Press, San Francisco, 2008.

[24] B.R. Soller, Design of intravascular fibre optic blood gas sensors, IEEE Eng. Med. Biol. 13 (1994) $327-335$. 


\section{Biographies}

Vincenzo Fabio Curto studied chemical engineering at University of Palermo, Italy (MSc Hons 2010). In 2010 he joined the Adaptive Sensors Group at Dublin City University where she is currently pursuing a PhD degree under the supervision of Prof. Dermot Diamond and Dr. Fernando Benito-Lopez. His research interests include the development of wearable micro-fluidic system to perform real-time analysis.

Shirley Coyle is a researcher/designer in the field of wearable technologies and smart textiles. She has combined expertise in Biomedical Engineering and Fashion Design. She received her BEng in Electronic Engineering in 2000 from Dublin City University, Ireland. She then worked in the Information and Communications division in Siemens Ltd. for 2 years before commencing a Ph.D. study to develop the first optical brain computer interface. She received her PhD from the National University of Ireland Maynooth in 2005. Studying by night she graduated from the Grafton Academy of Fashion Design in 2008. She has worked on the EU FP6 'Biotex' project, a European-wide multi-partner research effort to merge sensing capabilities with fabrics and textiles. She currently works within CLARITY: Centre for Sensor Web Technologies investigating ways to improve personal health and fitness using textile technologies.

Fernando Benito López studied chemistry at the Universidad Autonoma de Madrid and completed his master studies in the Department of Inorganic Chemistry in 2002. He obtained his PhD at the University of Twente, The Netherlands, under the supervision of Prof. David N. Reinhoudt and Dr. Willem Verboom in 2007. He carried out his postdoctoral research in the group of Prof. Dermot Diamond at Dublin City University, Dublin, Ireland. From 2010, he is Team Leader in polymer microfluidics at CLARITY: Centre for Sensor Web Technology, National Centre for Sensor Research, Dublin City University.

Robert Byrne studied pure and applied chemistry at Dublin City University (BSc Hons 2004) and received his PhD from Dublin City University (Materials Chemistry, 2008). His research interests include controlling molecular actuation by external stimuli for biomimetic applications.

Dermot Diamond received his PhD and DSc from Queen's University Belfast (Chemical Sensors, 1987, Internet Scale Sensing, 2002), and was VP for Research at Dublin City University (2002-2004). He has published over 200 peer-reviewed papers in international journals, is a named inventor in 13 patents, and is co-author and editor of three books. He is currently director of the National Centre for Sensor Research (www.ncsr.ie) and a Principle Investigator in CLARITY (www.clarity-centre.com/), a major research initiative focused on wireless sensor networks. In 2002 he was awarded the inaugural silver medal for Sensor Research by the RSC, London.

*Corresponding author: Tel.: +353 17007603 fax: +3531 7007995 .

E-mail addresses: fernando.lopez@dcu.ie (F. Benito-Lopez),

vincenzo.curto2@mail.dcu.ie (V.F. Curto),shirley.coile@dcu.ie (S. Coyle), robert.byrne@intel.com(R. Byrne), dermot.diamond@dcu.ie (D. Diamond). 


\section{Figure Captions}

Fig. 1. First generation of the micro-fluidic chip (a). Schematic representation of the fabrication steps for first and second generation of the micro-fluidic chip (b). Second generation of the micro-fluidic chip (c).

Fig. 2. Circuit diagram of the detection system in transmittance mode.

Fig. 3. Development of the electronics in the autonomous wearable micro-fluidic platform for sweat $\mathrm{pH}$ analysis. Textile-based fluid handling device (a), Wired detection system 2 (b), Wireless detection system (c) and miniaturised wearable micro-fluidic detector (d).

Fig. 4. Schematic representation of the alignment of LED-LED detector system in textile-based fluid handling device (a), and the alignment of SMD LED and light photo sensor in the transmittance detectors (b).

Fig. 5. Relation between channel length and the time that sweat needs to reach the sensing area using the passive pump in the chip (left) $(\mathrm{n}=5)$, picture of four micro-fluidic devices with different channel length (right)

Fig. 6. Average of the pumping rate of the passive pumping system, $n=4$.

Table 1. Basic/acidic delay and response times of the micro-fluidic chip

Fig. 7. Dynamic response of the micro-fluidic chip when alternatively is exposed to $\mathrm{pH} 1$ and $\mathrm{pH} 12$ solutions.

Fig. 8. Calibration curve of the fabric textile immobilised BCP dye.

Fig. 9. $\mathrm{pH}$ of sweat data recorded during 55 minutes of cycling exercise using the wireless detector and the micro-fluidic chip containing the thread. 
Table(s)

\begin{tabular}{l|c|c|c|c}
\multicolumn{1}{l}{} & Basic $\mathrm{t}_{\mathrm{d}} / \mathrm{min}$ & Acidic $\mathrm{t}_{\mathrm{d}} / \mathrm{min}$ & Basic $\mathrm{t}_{\mathrm{r}} / \mathrm{min}$ & Acidic $\mathrm{t}_{\mathrm{r}} / \mathrm{min}$ \\
\cline { 2 - 5 } Cycle 1 & 4.74 & 0.60 & 4.96 & 1.7 \\
Cycle 2 & 1.77 & 0.69 & 3.21 & 2.28 \\
Cycle 3 & 2.37 & 1.13 & 3.97 & 3.06 \\
Cycle 4 & 3.70 & 1.29 & 5.30 & 3.17
\end{tabular}

